\title{
Takotsubo Syndrome: Optimizing Care with a Multidisciplinary Approach
}

\author{
Ryuta Kinno (D) \\ Kenjiro Ono (iD ${ }^{2}$ \\ 'Division of Neurology, Department of \\ Internal Medicine, Showa University \\ Northern Yokohama Hospital, Yokohama \\ City, Kanagawa, 224-8503, Japan; \\ ${ }^{2}$ Division of Neurology, Department of \\ Medicine, Showa University School of \\ Medicine, Tokyo, 142-8555, Japan
}

\begin{abstract}
Symptoms of takotsubo syndrome (TTS) include acute and transient regional systolic dysfunction of the left ventricle, as well as a variety of wall-motion abnormalities. The clinical features of TTS, including initial symptoms, cardiac biomarkers, and electrocardiogram (ECG) changes, are similar to those of acute coronary syndrome, with the exception that TTS patients typically have no obstructive coronary artery disease. TTS primarily affects elderly women, and emotional or physical stress is a common cause of the disease. Exaggerated sympathetic stimulation associated with dysfunction of the limbic system has also been reported to be related to TTS occurrence. Cancer also induces emotional and physical stress. Therefore, optimization of TTS care should involve cardiac, neurological, psychiatric, and oncological approaches. The first step in optimizing TTS care is to diagnose it by cardiac means. Multimodality imaging, including ECG, echocardiogram, angiography, ventriculography, and cardiac magnetic resonance imaging, is indispensable for diagnosis, therapy management, and the evaluation of prognosis in the acute and chronic phases of TTS. The current cardiac approach during the acute phase is primarily supportive, with the goal of preventing life-threatening complications. As central nervous system diseases frequently trigger TTS, a neurological approach is also required. Appropriate psychiatric medication may reduce the risk of TTS recurrence, as not only psychiatric disorders themselves but also psychiatric medications can be the trigger for TTS. Several conditions are associated with TTS, including the novel coronavirus disease 2019. We present current knowledge of TTS in this review and describe how to optimize TTS care through a multidisciplinary approach.
\end{abstract}

Keywords: cardiac approach, neurological approach, psychiatric approach, oncological approach, limbic system, exaggerated sympathetic stimulation

\section{Introduction}

Takotsubo syndrome (TTS) was first described by Sato et al in $1990^{1}$ and has also been called stress cardiomyopathy, broken heart syndrome, apical ballooning syndrome, left ventricle (LV) apical ballooning syndrome, Takotsubo cardiomyopathy, and transient apical ballooning syndrome. The name derives from the Japanese fishing pot "Takotsubo," which is used to trap octopuses and has a form similar to the characteristic ballooning of the LV apex. Symptoms of TTS include acute and transient regional systolic dysfunction of the $\mathrm{LV}$, as well as a variety of wall-motion abnormalities (WMAs). ${ }^{2}$ The prognosis is generally considered to be favorable.

Although the clinical features-including initial symptoms, cardiac biomarkers, and electrocardiogram (ECG) changes - resemble those of acute coronary syndrome (ACS), there is no obvious obstructive coronary artery disease
Division of Neurology, Department of Internal Medicine, Showa University Northern Yokohama Hospital, 35-I

Chigasakichuo, Tsuzuki-ku, Yokohama

City, Kanagawa, 224-8503, Japan

Tel +8I-45-949-7000

Fax +8I-45-949-7293

Email kinno@med.showa-u.ac.jp 
explaining the severity of the LV systolic and diastolic dysfunction. ECG and/or left ventriculography are commonly used to detect transient WMAs. The syndrome primarily affects elderly women. Emotional or physical stress $^{3}$ and central nervous system (CNS) diseases are common triggers. ${ }^{4}$ Although the etiology of TTS remains unclear, exaggerated sympathetic stimulation, in association with dysfunction of the limbic system, has been reported to be related to TTS occurrence. Cancer also induces emotional and physical stress and a recent study demonstrated the strong association between TTS and malignant diseases. ${ }^{5}$ Therefore, TTS care optimization should involve cardiac, neurological, psychiatric, and oncological approaches. We present the current state of TTS knowledge and describe a multidisciplinary approach to optimize TTS care.

\section{Epidemiology}

A 2015 study reported that approximately 2\% of patients initially suspected as having ACS in fact had TTS, and most of these cases were postmenopausal women aged $\geq 50$ years. ${ }^{6}$ Another study found that up to $10 \%$ of female patients with TTS were initially suspected of having ACS. ${ }^{7}$ Schneider et al reported that approximately $90 \%$ of patients with TTS were middle-aged women, and approximately $80 \%$ were aged above $50 .{ }^{8}$ However, a more recent study by Topal et al concluded that TTS affects adults, children, and newborns alike, with no discernible sex differences. ${ }^{9}$ On the other hand, Templin et al reported that the etiology of TTS in women is more likely to involve emotional stress, while that in men is more likely to involve physical stress. ${ }^{2}$ Compared with younger patients, elderly patients with TTS tend to show poor outcomes caused by stroke, severe arrhythmias, acute heart failure, and cardiogenic shock. ${ }^{10}$ Moreover, many younger patients have nonapical anatomical variants and severe LV dysfunction. ${ }^{11}$

Singh et al discovered that TTS recurs within 6 months in $1.2 \%$ of patients and within 6 years in nearly $5 \%{ }^{12}$ ElBattrawy et al analyzed the data from a multicenter registry and found that the TTS recurrence rate was approximately $4 \% .{ }^{13}$ Kato et al analyzed the International Takotsubo (InterTAK) Registry and concluded that neurological and psychiatric diseases are predictors of TTS recurrence. ${ }^{14}$ Other possible predictors of TTS recurrence include hypertension ${ }^{13}$ and chronic obstructive pulmonary disease $(\mathrm{COPD})^{15}$ are also possible predictors of TTS recurrence.

\section{Pathogenic Mechanisms}

Although the precise pathophysiological mechanisms of TTS are unknown, significant evidence suggests exaggerated sympathetic stimulation is involved. ${ }^{16}$ TTS is frequently preceded by physical and emotional stress. ${ }^{2}$ Causes of catecholamine excess, such as pheochromocytoma, ${ }^{17} \mathrm{CNS}$ disorders,${ }^{18}$ and hyperactivity in specific cerebral regions, have been associated with this disease. ${ }^{19}$ In addition, intravenous catecholamine $\beta$-receptor agonist administration can cause TTS symptoms and various ballooning patterns. ${ }^{20} \mathrm{~A}$ previous study showed that the catecholamine levels are more elevated in patients with TTS triggered by emotional stress than in patients with myocardial infarction (MI). ${ }^{21}$ In addition, Kume et al discovered that patients with TTS have high norepinephrine levels in the coronary sinus, which would reflect an increase of myocardial catecholamines. ${ }^{22}$ In a study by Ortak et al, the cardiac parasympathetic activity markedly declined in patients with acute phase TTS, followed by autonomic modulation recovery between the subacute and chronic phases. ${ }^{23}$ According to a previous study with singlephoton emission computed tomography (SPECT), patients with TTS showed cardiac uptake of the ${ }^{123} \mathrm{I}-$ metaiodobenzylguanidine (MIBG) uptake in the akinetic LV apex, with almost normal perfusion within this region assessed by the ${ }^{99 \mathrm{~m}} \mathrm{Tc}-\mathrm{MIBI}$ SPECT. ${ }^{24}$ Therefore, exaggerated sympathetic stimulation may have a great impact on the pathogenic mechanisms of TTS.

As mentioned above, exaggerated sympathetic stimulation is crucial for the pathogenic mechanisms of TTS. However, the mechanism by which exaggerated sympathetic stimulation causes myocardial stunning in various regional ballooning patterns that characterize TTS remains unclear. Researchers have proposed several hypotheses, such as activation of myocardial survival pathways, catecholamine toxicity on cardiomyocytes, microcirculatory dysfunction, multivessel epicardial spasm, inflammation, and plaque rupture. ${ }^{16}$ Considering the high occurrence rate of TTS in postmenopausal females, estrogen deficiency may be an important factor for the pathophysiology of TTS. Previous studies showed that estrogens reduce mental stress ${ }^{25}$ and catecholamine-induced vasoconstriction. ${ }^{26}$ However, there were no evidence of correlation between estrogen levels and TTS. ${ }^{27}$ Clearly, further studies will be needed to elucidate the etiology of TTS. 


\section{Optimizing Care with a Multidisciplinary Approach Optimizing Care with a Cardiac Approach}

The first step in optimizing care is to diagnose TTS accurately via a cardiac approach. Multimodality imaging, including ECG, echocardiogram, angiography, ventriculography, and cardiac magnetic resonance (CMR) imaging, is indispensable for accurate diagnosis, management of therapy, and evaluation of prognosis. The diagnosis of TTS should be comprehensive; it should include diagnostic criteria and multimodality imaging.

\section{ECG Findings}

Increased heart rate, ST-segment elevation, and long QTc are the common ECG findings of TTS. ${ }^{2}$ Kurisu et al examined the temporal features of ECG of patients with $\mathrm{TTS}^{28}$ and reported the following ECG features: (1) the normalization of ST-segment changes within 3 days; (2) the persistence of ECG changes; (3) the similarity of temporal features of the ECG to those of patients with minimal acute MI (AMI), and (4) patients with TTS showed deeper T-wave inversion and a longer QTc interval than patients with the minimal AMI group during the acute phase. Thus, the temporal changes in ECG in patients with TTS resemble those in patients with AMI. Physicians must take care to differentiate these cardiac disorders for effective patient management.

\section{Biomarkers}

Two types of biomarkers, namely, natriuretic peptides and cardiac myonecrosis markers, are commonly used for TTS diagnosis. At the time of diagnosis, the levels of plasma B-type natriuretic peptide $(\mathrm{BNP})^{29}$ or its precursor N-terminal pro-BNP (NT-proBNP) ${ }^{30}$ are often high in patients with TTS. In contrast, patients with TTS typically show lower levels of the cardiac myonecrosis biomarkers cardiac troponin and creatine kinase-MB (CKMB) than patients with AMI, as tissue necrosis is mild or absent in patients with TTS. ${ }^{31}$ Relations between these biomarkers, including the troponin $\mathrm{T} / \mathrm{CKMB}$ ratio, ${ }^{32} \mathrm{BNP} /$ troponin I ratio, ${ }^{33}$ and NT-pro-BNP/troponin $\mathrm{T}$ ratio, ${ }^{34}$ may be useful for the differential diagnosis of TTS. However, in some cases, there are no significant differences in troponin levels between patients with TTS and patients with AMI. Moreover, in TTS, the degree of angiographic myocardial dysfunction differs from the extent of biomarker elevation. ${ }^{35}$ Several other novel markers, such as copeptin, ischemic modified albumin, soluble lectin-like oxidized low density lipoprot receptor-1, and soluble suppression of tumorigenicity-2, may be possible biomarkers for TTS. $^{36}$

\section{Echocardiography}

Most patients with suspected TTS initially undergo a transthoracic echocardiogram. ${ }^{37}$ This helps to detect the apical-midventricular ballooning and the WMA patterns. The echocardiogram also provides helpful information about contractile function and complications, including LV outflow tract obstruction (LVOTO; intraventricular gradient $>25 \mathrm{mmHg}$ ), LV thrombi, pericardial effusion, reversible moderate-to-severe mitral regurgitation, and right ventricular (RV) involvement. The RV involvement, though transient, leads to longer hospitalization and hemodynamic instability. ${ }^{38}$

Contrast echocardiography is used to obtain accurate information on wall motion (apical, midventricular, or basal ballooning) and the existence of intraventricular thrombus in patients with a poor acoustic window. ${ }^{37}$ In addition, 3D echocardiography is useful for quantifying chamber measurements, with higher accuracy and reproducibility than 2D echocardiography. ${ }^{39}$ Compared with 2D echocardiography, 3D echocardiography provides more detailed information regarding the regional WMAs. ${ }^{40}$ Moreover, 3D echocardiography may be useful for detecting the RV involvement, even in the acute phase. Finally, strain imaging using speckle tracking echocardiography may help assess the severity of RV involvement. ${ }^{41}$

\section{Angiography and Ventriculography}

As mentioned above, differentiating TTS from AMI is difficult, as the two conditions have similar clinical features, including the clinical course, ECG, and cardiac biomarkers. ${ }^{42}$ Compared to patients with AMI, most patients with TTS have higher troponin levels but similar ECG findings. ${ }^{2}$ Moreover, patients with TTS frequently have coronary artery disease; this coexistence often leads to adverse outcomes, ${ }^{43}$ but the coronary arteries of these patients often show almost normal findings. Therefore, early examination of the coronary arteries by angiography is important in order to exclude the possibility of ACS.

Echocardiography and left ventriculography are useful in assessing regional WMAs. ${ }^{16}$ All patients with suspected TTS should have LV ventriculography, since this modality can be used to diagnose TTS during cardiac catheterization. ${ }^{37}$ Biplane left ventriculography can assess 
LV function and type of WMA, including by differentiating four morphological patterns of TTS, ie, apical TTS, midventricular TTS, basal TTS, and focal TTS. ${ }^{37,44}$

\section{CMR}

CMR is used for the extensive assessment of structural and functional alterations of cardiac tissues of patients with TTS. $^{45}$ There are three CMR diagnostic criteria for TTS: conventional regional WMAs; the existence of reversible tissue injury (edema); and the absence of irreversible tissue injury (late gadolinium enhancement) when signal intensity thresholds of 5 standard deviations are applied.

The further merit of CMR is that it provides useful information regarding the differential diagnosis (MI and myocarditis), it provides pathophysiological information, and it can detect complications (eg, LV thrombi) for the managements of patients with TTS. CMR should be performed in the acute phase for patients with suspected TTS. In particular, it should be done if another type of MI with nonobstructive coronary arteries (eg, myocarditis) is suspected, and a different cardiac approach is required. In the chronic phase, all patients with TTS should be examined by CMR within 2 months, especially if abnormalities of ECG and/or echocardiographic findings persist, because such clinical courses are not typical of TTS.

\section{Diagnostic Criteria for TTS}

Currently, no single universally accepted diagnostic criteria are available for TTS; thus, several criteria have been proposed for diagnosing TTS. Among these criteria, the Mayo Clinic diagnostic criteria are perhaps the most wellestablished (Box 1). ${ }^{42}$ The Heart Failure Association diagnostic criteria for TTS are also well known (Box 2). ${ }^{46,47}$ Another set of criteria, the InterTAK diagnostic criteria ${ }^{27}$ incorporate several different aspects of TTS as follows: (i) $\mathrm{RV}$ and other atypical WMAs are involved; (ii) TTS is not necessarily accompanied by emotional or physical stress; (iii) potential triggers for TTS are neurological disorders and pheochromocytoma; and (iv) the coexistence of coronary artery disorder and TTS has been established (Box 3).

Moreover, the InterTAK Diagnostic Score is a clinical scoring method used to assess the probability of TTS and to differentiate TTS from ACS in the acute phase. ${ }^{48}$ Based on the ECG findings and clinical course; this score consists of the following indices: female sex (25 points); emotional stress (24 points); physical stress (13 points); absence of ST-segment depression (12 points); acute,

\section{Box I Mayo Clinic Criteria for TTS}

I. Transient hypokinesis, akinesis, or dyskinesis of the LV midsegments with or without apical involvement; the regional WMAs extend beyond a single epicardial vascular distribution; a stressful trigger is often but not always present.

2. Absence of obstructive coronary disease or angiographic evidence of acute plaque rupture.

3. New ECG abnormalities (either ST-segment elevation and/or T-wave inversion) or modest elevation in cardiac troponin.

4. Absence of pheochromocytoma and myocarditis.

Notes: Reprinted from Am Heart J. 155(3), Prasad A, Lerman A, Rihal CS. Apical ballooning syndrome (Tako-Tsubo or stress cardiomyopathy): a mimic of acute myocardial infarction. Am Heart J. 2008;155(3):408-417, Copyright (2008), with permission from Elsevier. ${ }^{42}$

Abbreviations: ACS, acute coronary syndrome; ECG, electrocardiography; LV, left ventricular, TTS, Takotsubo syndrome; WMA, wall motion abnormality.

Box 2 Heart Failure Association Diagnostic Criteria for TTS

I. Transient regional WMAs of the LV or RV myocardium that are frequently, but not always, preceded by a stressful trigger (emotional or physical).

2. The regional WMAs usually extend beyond a single epicardial vascular distribution and often result in circumferential dysfunction of the ventricular segments involved.

3. Absence of atherosclerotic coronary artery diseases including acute plaque rupture, thrombus formation, and coronary dissection or other pathological conditions, that explain the pattern of temporary LV dysfunction observed (eg, hypertrophic cardiomyopathy, viral myocarditis).

4. New and reversible ECG abnormalities (ST-segment elevation, ST depression, LBBB, T-wave inversion, and/or long QTc) occur during the acute phase ( 3 months).

5. Serum BNP or NT-proBNP levels are significantly elevated during the acute phase.

6. Cardiac troponin measured by a conventional assay is positive but at a relatively small elevation (ie, disparity between the troponin level and the amount of dysfunctional myocardium present).

7. Recovery of ventricular systolic function on cardiac imaging at follow-up (3-6 months).

Notes: Reprinted from Lyon AR, Bossone E, Schneider B, et al. Current state of knowledge on Takotsubo syndrome: a Position Statement from the Taskforce on Takotsubo Syndrome of the Heart Failure Association of the European Society of Cardiology. Eur J Heart Fail. 2016;18(I):8-27. @ 2015 The Authors European Journal of Heart Failure $\odot 2015$ European Society of Cardiology. ${ }^{46}$

Abbreviations: ECG, electrocardiography; LBBB, left bundle branch block; LV, left ventricle; RV, right ventricle; TTS, Takotsubo syndrome; WMA, wall motion abnormality. 
Box 3 International Takotsubo Diagnostic Criteria (InterTAK Diagnostic Criteria)

I. Patients show transient LV dysfunction (hypokinesia, akinesia, or dyskinesia) presenting as apical ballooning or midventricular, basal, or focal WMAs. RV involvement can be present. Aside from these regional wall-motion patterns, transitions between all types can exist. The regional WMA usually extends beyond a single epicardial vascular distribution; however, rare cases can exist wherein the regional WMA is present in the subtended myocardial territory of a single coronary artery (focal TTS).

2. An emotional, physical, or combined stress can precede the TTS event but is not obligatory.

3. Neurological disorders as well as pheochromocytoma may serve as triggers of TTS.

4. New ECG abnormalities are present (ST-segment elevation, STsegment depression, T-wave inversion, and QTc prolongation); however, rare cases exist without any ECG changes.

5. The levels of cardiac biomarkers (troponin and creatine kinase) are moderately elevated in most cases; significant elevation of brain natriuretic peptide is common.

6. Significant coronary artery disease is not a contradiction in TTS.

7. Patients have no evidence of infectious myocarditis.

8. Postmenopausal women are predominantly affected.

Note: Reprinted from Ghadri JR, Wittstein IS, Prasad A, et al. International Expert Consensus Document on Takotsubo Syndrome (Part I): clinical Characteristics, Diagnostic Criteria, and Pathophysiology. Eur Heart J. 2018;39(22):2032-2046. Creative Commons license and disclaimer available from http://creativecommons. org/licenses/by-nc/4.0/. ${ }^{27}$

Abbreviations: ECG, electrocardiography; TTS, Takotsubo syndrome; WMAs, wall motion abnormalities.

former, or chronic psychiatric disorder (11 points); acute, former, or chronic neurological disorders (9 points); and long QTc (6 points). Patients with InterTAK scores of $\geq 50$ or $\leq 31$ were almost always correctly diagnosed as having TTS or ACS, respectively. ${ }^{48}$

\section{Recommended Cardiac Approach for Acute-Phase TTS}

Although TTS is considered benign, $21.8 \%$ of patients develop serious complications in the hospital. ${ }^{2}$ Kato et al demonstrated that $40 \%$ of patients have in-hospital cardiac complications, including pulmonary edema and cardiogenic shock. ${ }^{49}$ The current cardiac approach during the acute phase is thus primarily supportive, with the goal of preventing life-threatening complications. $^{50}$ Patients with long QTc should undergo ECG monitoring because they are at high risk for ventricular arrhythmias; ${ }^{51}$ therefore, ECG monitoring for at least one day is mandatory for all patients with TTS. Diagnosed TTS patients should be given supportive pharmacological treatment (eg, $\beta$ blockers, diuretics, anticoagulants, antiarrhythmics, and noncatecholamine inotropes such as levosimendan). Nonpharmacological support may include mechanical circulatory and respiratory support, depending on the grade of heart failure and concomitant complications. Among forms of mechanical circulatory support, intraaortic balloon pumps should not be used unless LVOTO has been ruled out.

If hemodynamically significant LVOTO is present, there may be hypercontractility of the basal segments, in which case inotropic drugs should be immediately stopped in order to avoid further obstruction. Short-acting intravenous $\beta$-blockers should be considered when patients have no severe heart failure. Esmolol, a cardioselective-1 blocker with a short half-life (9 minutes), may be a simple treatment option for patients with TTS and LVOTO. Santoro et al found in a case series of 10 patients with TTS and LVOTO that esmolol infusion ameliorated the reductions in intraventricular gradient and systemic blood pressure. ${ }^{52}$ Experimental data showed a highdensity of $\beta 2$-adrenergic receptors in the apical and midventricular segments of the LV, and a high-density of $\beta 1$-adrenergic receptors in the basal LV segments. ${ }^{53}$

Conversely, when there is cardiogenic shock without LVOTO, catecholamines (ie, inotropes) can be used to maintain pressure. Levosimendan, a catecholamine-sparing positive inotrope, may be a useful alternative, ${ }^{54}$ although there is limited evidence supporting its use. Mechanical support should be considered early in the treatment of patients whose cardiac output is low. A microaxial blood pump may help as it has the advantage of not increasing the afterload. $^{2}$ If there are no contraindications, patients with refractory shock may require extracorporeal life support or a temporary LV assist device. The use of standard cardiac approaches to reduce preload in TTS patients with congestive heart failure (eg, diuretics and nitroglycerine) should be carefully considered. There is no positive evidence to support the routine use of $\beta$-blockers for the management of TTS during the acute phase. ${ }^{55}$

A serious complication of TTS is intraventricular thrombus. It is seen in patients with severe WMAs and low LV ejection fraction (LVEF); ${ }^{45,56}$ nearly $25 \%$ of patients with TTS suffer from this complication. Prophylactic anticoagulation should be considered for all patients with TTS to prevent LV apical thrombus 
Table I Important Signs in TTS Patients Indicating a Need for Consultation with Specialists

\begin{tabular}{|l|l|l|}
\hline $\begin{array}{l}\text { Specialist } \\
\text { Consulted }\end{array}$ & Acute Phase & Chronic Phase \\
\hline Neurologist & Acute stroke symptoms; Seizure & Memory deficits \\
\hline Psychiatrist & $\begin{array}{l}\text { Preexisting psychiatric illness; Taking psychiatric } \\
\text { medication }\end{array}$ & $\begin{array}{l}\text { No apparent physical and emotional stress before onset; Psychiatric } \\
\text { symptoms }\end{array}$ \\
\hline Oncologist & Past history of chemotherapy & Positive for screening test of cancer \\
\hline Other specialists & COVID-19 & Remarkable past history \\
\hline
\end{tabular}

Notes: Remarkable past history includes COPD, asthma, a pulmonary circulatory disorder, hyper- or hypothyroidism, alcohol abuse, drug abuse, surgery, chronic connective tissue disease, chronic liver disease, chronic kidney disease, sexual intercourse, ${ }^{98}$ and pregnancy.

Abbreviation: COVID-19, coronavirus disease 2019.

formation. ${ }^{57,58} \mathrm{~A}$ recent study demonstrated that the InterTAK Thrombus Risk Score (Table 1) is a useful tool to assess the risk for thrombus formation for patients with TTS. ${ }^{59}$ This score consists of the following indices: apical type TTS (1.5 points); previous vascular disease (1.5 points); LVEF $\leq 30 \%$ on admission (1.5 points); and white blood cell counts on admission $>10,000$ cells $/ \mu \mathrm{L}$ (1 point). Patients with an InterTAK Thrombus Risk Score of $\geq 3$ are considered to be at high risk of thrombus formation.

Madias et al reported that nearly $9 \%$ of their patients with TTS had torsades de pointes or ventricular fibrillation, and long QTc was significantly correlated with ventricular arrhythmia occurrence. ${ }^{51}$ Temporary cardiac pacing at a high rate ${ }^{60}$ or intravenous magnesium ${ }^{61}$ has been reported to be effective in preventing ventricular fibrillation recurrence. The need for an implantable cardioverter-defibrillator (ICD) for ventricular arrhythmia prevention in the acute phase remains unconfirmed; however, considering the reversible nature of TTS, ICD therapy may not be required.

\section{Recommended Cardiac Approach for Chronic-Phase TTS}

To date, the effectiveness of renin-angiotensin system inhibitors (RASi) such as angiotensin-converting enzyme inhibitors or angiotensin II receptor blockers remains controversial. Templin et al showed that the use of RASi improved survival during the chronic phase. ${ }^{2}$ In a meta-analysis by Singh et al, some of the studies found that RASi showed benefit. ${ }^{12}$ These findings could be explained by a decrease in sympathetic activity via the RAS or an anti-inflammatory effect on the myocardium. On the other hand, Santoro et al found that RASi had no benefit, ${ }^{62}$ while Brunetti et al showed that RASi had benefit only when associated with a $\beta$-blocker. ${ }^{63}$ Recent multicenter studies showed that RASi had no benefit ${ }^{13,14}$ even when used in combination with a $\beta$-blocker. ${ }^{15}$

In addition, $\beta$-blockers may help to protect against catecholamine surges following stressful triggers. Indeed, $\beta$-blockers are a common discharge medication for TTS patients. However, there is no conclusive evidence regarding the effectiveness of $\beta$-blockers on mortality in the chronic-phase. $^{2}$ Two meta-analyses also showed that $\beta$ blockers had no preventive effect against TTS recurrence. ${ }^{12,62}$ Hence, the effects of $\beta$-blockers during the chronic-phase might be more restrictive than expected.

In regard to the pathogenesis of TTS, the idea of using estrogen supplements to prevent recurrence is an intriguing one. However, no studies have investigated this. At the present time, estrogen supplementation is not recommended, as there is an increased risk for venous thromboembolism.

\section{Optimizing Care with a Neurological Approach}

TTS is frequently triggered by CNS diseases. ${ }^{4}$ Ischemic stroke, subarachnoid hemorrhage, and seizures are the bestknown causes of TTS. ${ }^{16}$ Meanwhile, amyotrophic lateral sclerosis, encephalopathy syndrome, intracerebral bleeding, migraine, posterior reversible encephalitis/meningitis, and traumatic injury are rare triggers of TTS. ${ }^{4}$ In one case report, TTS was triggered by a possible limbic encephalopathy caused by high-grade B-cell lymphoma. ${ }^{64}$ We have also recently reported a patient with TTS associated with autonomic limbic encephalitis. ${ }^{65}$ Thus, neurological disorders are clearly associated with TTS occurrence.

\section{Dysfunction of the Limbic System}

While there is strong evidence to support the involvement of the brain-heart axis in TTS, ${ }^{66}$ the pathological mechanism of 
A

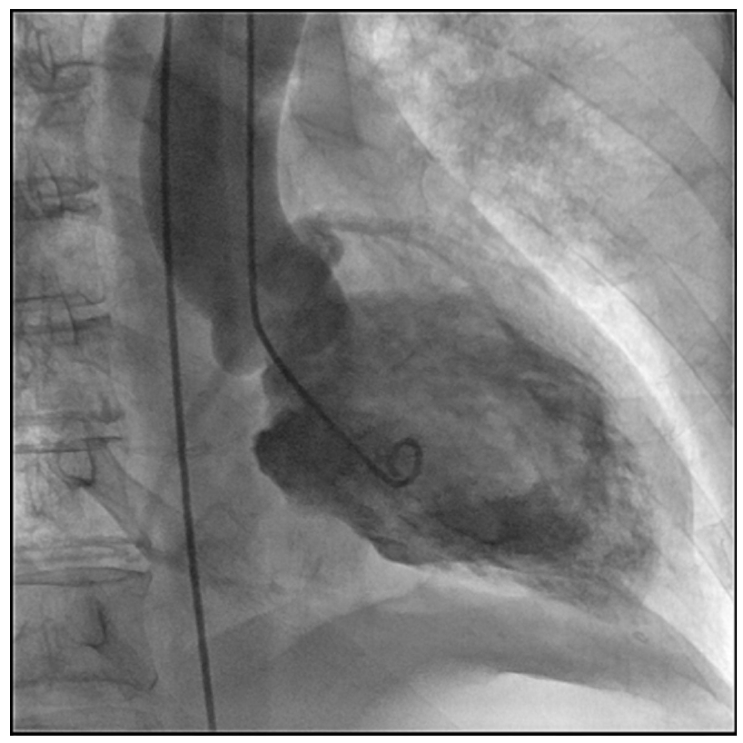

B

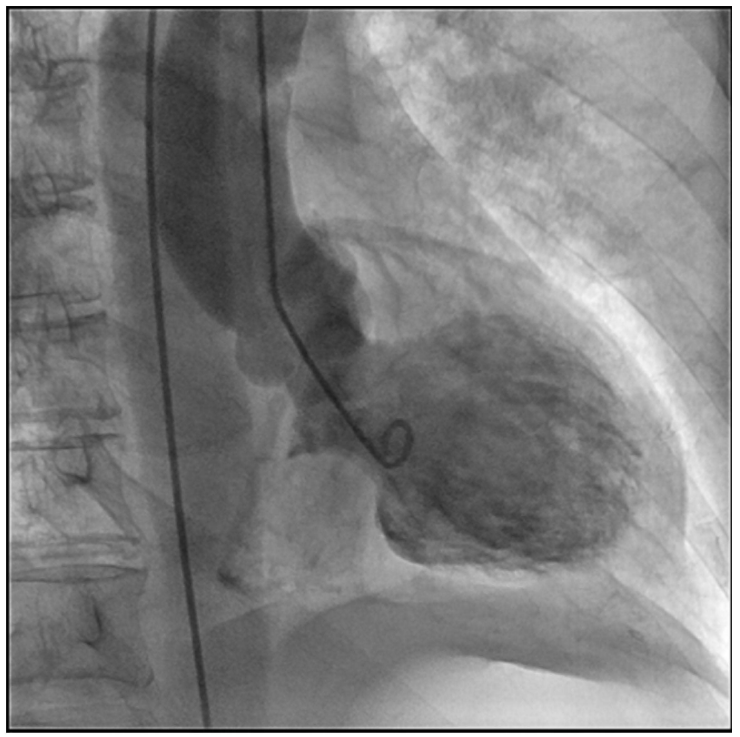

C

Before treatment

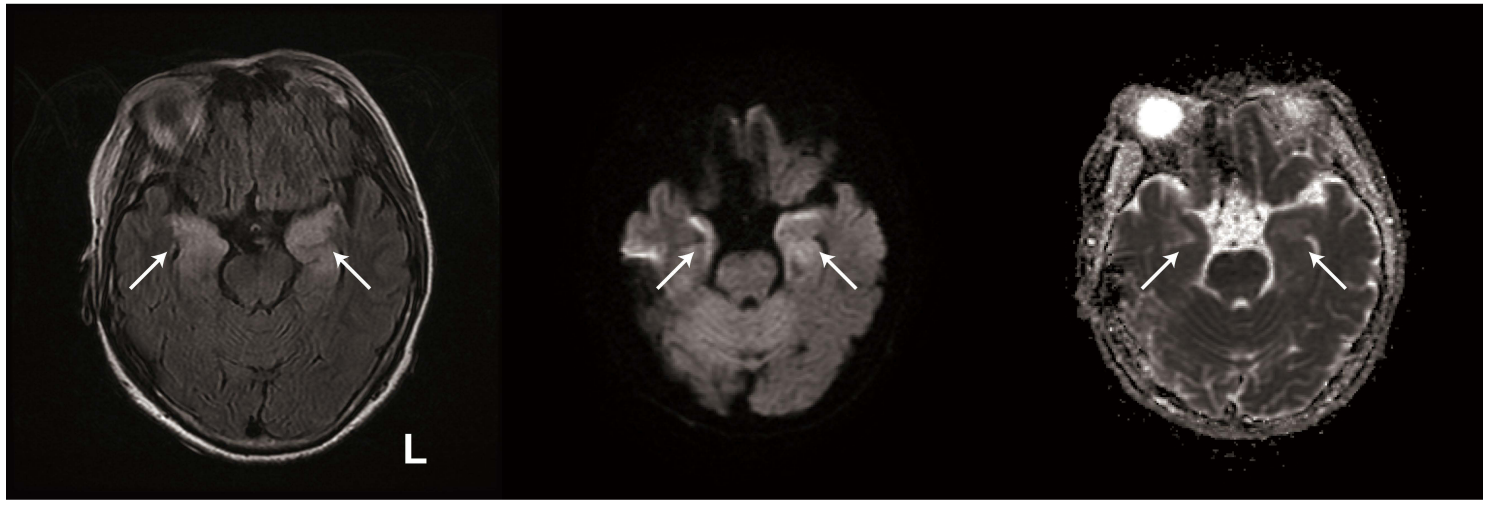

D

After treatment

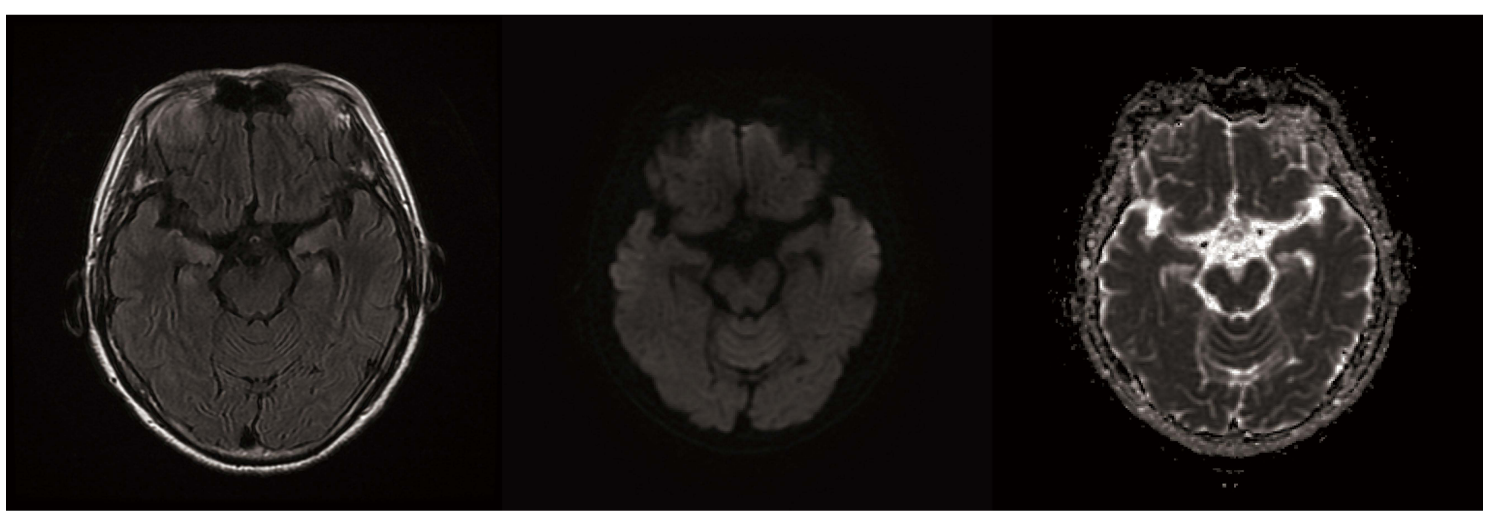

Figure I TTS following autoimmune limbic encephalitis. (A and B) Left ventriculographic findings. The diastole (A) and systole (B) images are shown. (C and D) MRI findings. The initial MRI (C) revealed abnormal hyperintense areas (arrows) in the bilateral medial temporal lobes on the fluid-attenuated inversion recovery image (left). Diffusion-weighted imaging (middle) and an apparent diffusion coefficient map (right) both revealed abnormal hyperintensity in these areas. The abnormalities were all resolved in follow-up MRI (D).

Notes: Reproduced from Kakinuma Y, Kimura T, Sakae Y, Kubota S, Ono K, Kinno R. Takotsubo syndrome associated with autoimmune limbic encephalitis: a case report. BMC Cardiovasc Disord. 2021;2I(I):86. Creative Commons license and disclaimer available from: http://creativecommons.org/licenses/by/4.0/legalcode. ${ }^{65 .}$ Abbreviation: MRI, magnetic resonance imaging. 
TTS remains poorly understood. It has been hypothesized that exaggerated sympathetic stimulation plays a role in the etiology of TTS. ${ }^{21}$ The limbic system consists of several regions, such as the amygdala, cingulate cortex, hippocampus, and insula, and this system may have a role in regulating the autonomic nervous system. ${ }^{67}$ According to a functional magnetic resonance imaging (MRI) study, patients with TTS showed poor connectivity among the brain regions that are important for autonomic functions, including the limbic system. $^{68}$ Recently, a rare case of midventricular TTS (Figures 1A and B) was associated with autoimmune limbic encephalitis. ${ }^{65}$ Before the onset of TTS, the patient exhibited amnesia and topographical disorientation. Brain MRI revealed hyperintense lesions in the bilateral temporal regions, which were most visible during the fluidattenuated inversion recovery sequence (Figure 1C). Given these findings, it is possible that exaggerated sympathetic stimulation is caused by the limbic system dysfunction resulting from autoimmune limbic encephalitis.

The relationship between the occurrence of TTS and exaggerated sympathetic stimulation remains unclear; however, the spillover of stress-related neuropeptides may be associated with this relationship. ${ }^{69}$ A complex interaction between neocortical regions and the limbic system occurs in response to stress by activating brainstem noradrenergic neurons and stress-related neuropeptides (ie, the neuropeptide Y [NPY] produced by the arcuate nucleus in the hypothalamus). These neuropeptides are stocked in the presynaptic terminals of the postganglionic sympathetic system. When they spill at the myocardium through both microvascular dysfunction and/or direct catecholamine toxicity, myocardial stunning could occur. Patients with TTS due to CNS disease showed worse outcomes than those with TTS due to an emotional trigger. ${ }^{70,71}$ Thus, clarifying the role of limbic system diseases in TTS is clinically important. Further work is needed to confirm the link between the pathophysiology of TTS and the brain-heart axis.

\section{Recommended Neurological Approach for TTS}

As mentioned above, neurological disorders are clearly associated with TTS. Hence, the following neurological approaches are recommended for patients with TTS. ${ }^{18}$ First, every patient with ACS symptoms and an acute neurological disorder, such as subarachnoid hemorrhage, seizure, or ischemic stroke, should be thoroughly evaluated for TTS. The presence of acute stroke symptoms, including face drooping, arm weakness, and speech difficulty, should be checked in all patients with TTS (Table 1).
Echocardiography should be routinely performed in cases with any of the following conditions: cardiac symptoms that developed after a CNS event, the presence of ECG dynamics, elevation of CK or troponin, or an MI-like appearance. Second, blood MI parameters should be determined in all patients with CNS disease accompanied by cardiac symptoms, and a routine ECG should be considered regardless of the presence or absence of cardiac symptoms. CNS-triggered TTS must be recognized because appropriate treatment can improve the overall outcome of these patients. The underlying neurological disorder may also improve with adequate TTS treatment. In the chronic phase, all patients should be checked for their memory functions, as memory deficit is often overlooked in the acute phase and is possible sign of limbic encephalitis. If the patients have memory deficits, a neurologist should be consulted.

Moreover, appropriate treatment for neurological disorders may contribute to the management of TTS recurrence. For example, a patient with TTS and autoimmune limbic encephalitis was intravenously injected with methylprednisolone (1 g/day) for 5 days; subsequently, neurological symptoms, such as disorientation, and abnormal MRI findings gradually improved (2 months after admission; Figure 1D). ${ }^{65}$ The more CNS disorders that carry the potential to trigger TTS are detected, the more attention will be drawn to CNS-triggered TTS. TTS must be recognized and understood by neurologists not only to distinguish it from MI, but also to contribute to the positive outcome of these patients.

\section{Optimizing Care with a Psychiatric Approach}

One of the well-known triggers of TTS is emotional stress, such as the unanticipated death of a close relative. TTS can also be triggered by positive emotional experiences (for example, a positive job interview, a surprise farewell celebration, or becoming a grandmother); thus, it is also known as the "happy heart syndrome."72 Patients with TTS have a higher rate of psychiatric and neurological disorders than those with ACS. ${ }^{16}$ These associations have been extensively reported. Preexisting psychiatric illness and even acute exacerbations of psychiatric disorders or changes in psychotropic medications have been reported as triggers of TTS. ${ }^{73}$

\section{Psychiatric Association of TTS}

Psychiatric comorbidities, such as depression and anxiety, are more prevalent in TTS patients than those with ACS. ${ }^{74}$ Studies have found a link between TTS and anxiety disorders, mood disorders, adjustment disorders, the 
schizophrenia-spectrum, and neurocognitive and other psychotic disorders. ${ }^{2,73,75,76}$ Acute exacerbations of psychiatric disorders, such as anxiety disorders, depression, mania, or psychosis, have been reported as potential triggers of TTS. $^{75,77}$ In the International Takotsubo Registry, approximately $10 \%$ of patients had acute psychiatric disorders, with mood and anxiety disorders accounting for $4 \%$ and $1 \%$, respectively. ${ }^{2}$ Changes in psychiatric medications can also trigger TTS; according to multiple reports, cases have been linked to both the up-titration and overdose of serotoninnorepinephrine reuptake inhibitors. ${ }^{78-80}$ Lithium, used in patients with bipolar disorder, has been associated with elevated serum catecholamine levels and reported to trigger TTS. $^{81}$ Electroconvulsive therapy, used in patients with severe major depression or bipolar disorder, temporarily and abruptly raises serum catecholamine levels, and is linked to the occurrence of TTS. ${ }^{82}$ In consideration of all these findings, TTS is clearly linked to psychiatric disorders.

\section{Recommended Psychiatric Approach for TTS}

Patients with preexisting psychiatric illness have a higher risk of TTS recurrence, ${ }^{83}$ although the etiology is unknown. In addition, not only psychiatric disorders themselves but also the psychiatric medications used to treat them can be the trigger for TTS. Therefore, it is recommended that patients with TTS should be followed closely for the psychiatric symptoms (Table 1). First, if patients have a preexisting psychiatric illness and/or take psychiatric medication, the type and amount of drug should be determined, and if necessary, the psychiatrist should be consulted even in the acute phase. Second, if emotional stress is not apparent before the onset of TTS, a preexisting psychiatric illness should be considered, whether or not there is a history of one. Finally, TTS patients with psychiatric symptoms should have appropriate long-term management for psychiatric illness after recovering from their cardiac symptoms. Currently, there is little evidence of a benefit of psychiatric treatment in patients with TTS; however, psychiatric illness is also associated with increased mortality. Considering the clear association between TTS and psychiatric disorders, appropriate psychiatric medication may mitigate TTS recurrence.

\section{Optimizing Care with an Oncological Approach}

Patients with TTS have a high rate of malignancy. ${ }^{84}$ Desai et al demonstrated that the mean incidence of TTS is 53.0 per 100,000 hospitalized chemotherapy patients with no apparent sex differences. ${ }^{85}$ A recent study demonstrated clear associations between the worse outcome of TTS and cancer disease others than pheochromocytoma. ${ }^{5}$ Patients with cancer have not only physical but also emotional stress; both types of stress would be associated with the occurrence of TTS, as discussed above. ${ }^{86}$ Such patients are likely to experience multiple episodes of stress in relation to the initial diagnosis, the chronic inflammation of the tumor, and the various cancer treatments including chemotherapy and radiation therapy. In addition, the circulation of paraneoplastic mediators may modify the adrenoreceptors in cardiac tissue, leading to systolic dysfunction of LV. All these factors would affect the occurrence of TTS for tumor patients.

\section{Effects of Chemotherapy on TTS}

Not only the effects of the tumor itself but also the physical and psychological effects of the associated chemotherapy constitute possible risk factors for TTS. The associations between several types of chemotherapy and TTS have been reported previously. ${ }^{87}$ 5-Fluorouracil (5-FU) is commonly used in many cancers and can induce several side effects, including leukoencephalopathy, ${ }^{88}$ diarrhea, and gastrointestinal mucositis. ${ }^{89}$ 5-FU-induced TTS has been reported in the literature. ${ }^{90}$ Capecitabine, ${ }^{91}$ bevacizumab, ${ }^{92}$ combretastatin, ${ }^{93}$ rituximab, ${ }^{94}$ and tyrosine kinase inhibitors ${ }^{95}$ have also been reported as possible triggers of TTS. Immune checkpoint inhibitors including ipilimumab, pembrolizumab and nivolumab have come to be used for cancer treatments over the last ten years. There has also been a single case report of a patient with metastatic melanoma who showed a TTS-like syndrome with apical ballooning and hyperdynamic basal segment followed by the ipilimumab treatment. ${ }^{96}$ In all cases, the causality between chemotherapy and the occurrence of TTS remains unclear. Studies will be needed.

\section{Recommended Oncological Approach for TTS}

The effect of cancer treatment on the occurrence and clinical course of TTS remains unclear. However, considering the clear associations between TTS and cancer revealed in the previous studies, ${ }^{5,86}$ tumor screening should be performed for patients with TTS-like syndromes with apical ballooning, especially patients with no apparent neurological or psychiatric disorders (Table 1). On the other hand, patients who underwent chemotherapy for cancer should be followed closely for the presence of cardiac symptoms such as chest pain or breathing difficulty. Cardiac examinations including assays for biomarkers and imaging by ECG and echocardiogram would be 
helpful for immediate diagnosis of TTS. Chemotherapy can cause neurological disorders such as stroke or encephalopathy, ${ }^{88}$ which may be related to TTS. In addition, a psychological approach is also important for the management of psychiatric stress. Therefore, a multipronged approach including cardiac, neurological, psychological, and/or oncological methods is needed for the optimized care of these patients.

\section{Optimizing Care with Other Approaches}

Several conditions are associated with TTS occurrence. According to a previous systematic review, ${ }^{35} \mathrm{COPD}$, asthma, and a pulmonary circulatory disorder have been reported as comorbidities of TTS. Hyper- or hypothyroidism is another comorbidity. A recent study demonstrates that $7 \%$ of patients with TTS is related to acute pulmonary disease, and the presence of acute pulmonary disease is associated with a severe in-hospital course and a worse long-term outcome. $^{97}$ Small numbers of TTS patients have a history of alcohol abuse, drug abuse, or surgery. Chronic connective tissue disease, chronic liver disease, and chronic kidney disease have also been found in TTS cases. TTS may be triggered not only by severe medical conditions but also by physiological processes, such as sexual intercourse ${ }^{98}$ and pregnancy. ${ }^{99}$ Surprisingly, acute coronary artery obstruction can also cause TTS. ${ }^{100}$ Hence, the medical history of patients should be thoroughly examined. Any remarkable signs should be followed closely, and if necessary, specialists should be consulted in the chronic phase.

Presently, of course, the world is in the throes of a great pandemic caused by the severe acute respiratory syndrome coronavirus 2 (SARS-CoV-2) and associated novel coronavirus disease 2019 (COVID-19). SARS-CoV-2 infection has been linked to a number of cardiovascular complications, including arrhythmia, myocarditis, myocardial injury, and exacerbation of chronic cardiac diseases such as heart failure. $^{101-103}$ A recent study of 118 patients with COVID19 who had clinically indicated transthoracic echocardiogram (TTE) found that 5 of them were compatible with a TTS diagnosis. ${ }^{104}$ Thus, TTS should be considered in the differential diagnosis of COVID-19 patients with cardiovascular complications, although the diagnosis is difficult because cardiac imaging modalities need to be modified to minimize patient contact. ${ }^{105}$ Considering the severity of COVID-19, it is better that these patients are diagnosed in the acute phase. Thus, in patients with COVID-19 with cardiovascular complications, TTE evaluation may be useful to guide treatment and determine the need for further invasive workup.

\section{Conclusion}

Although TTS is recognized globally, its exact pathophysiological mechanisms remain unclear. TTS is not a benign condition. Neurological, psychiatric, and oncologic disorders, and even COVID-19, have been reported to be associated with TTS occurrence. Limbic system dysfunction may be a key factor in exaggerated sympathetic stimulation, which results in TTS. Therefore, a multidisciplinary approach, which includes cardiac, neurological, psychiatric, and oncological components, can be beneficial in managing TTS. Further research into the exact pathophysiology is required to improve therapeutic strategies for TTS.

\section{Acknowledgments}

We would like to express our gratitude to H. Kawano for her administrative assistance.

\section{Disclosure}

The authors report no conflicts of interest in this work.

\section{References}

1. Sato H, Tateishi H, Uchida T, et al. Clinical aspect of myocardial injury: from ischemia to heart failure. Kagaku Hyoronsha. 1990;2:55-64.

2. Templin C, Ghadri JR, Diekmann J, et al. Clinical Features and Outcomes of Takotsubo (Stress) Cardiomyopathy. $N$ Engl J Med. 2015;373(10):929-938. doi:10.1056/NEJMoa1406761

3. Tsuchihashi K, Ueshima K, Uchida T, et al. Transient left ventricular apical ballooning without coronary artery stenosis: a novel heart syndrome mimicking acute myocardial infarction. $J$ Am Coll Cardiol. 2001;38(1):11-18. doi:10.1016/S0735-1097(01)01316-X

4. Finsterer J, Wahbi K. CNS disease triggering Takotsubo stress cardiomyopathy. Int $J$ Cardiol. 2014;177(2):322-329. doi:10.1016/j.ijcard.2014.08.101

5. Sattler K, El-Battrawy I, Lang S, et al. Prevalence of cancer in Takotsubo cardiomyopathy: short and long-term outcome. Int J Cardiol. 2017;238:159-165. doi:10.1016/j.ijcard.2017.02.093

6. Akashi YJ, Nef HM, Lyon AR. Epidemiology and pathophysiology of Takotsubo syndrome. Nat Rev Cardiol. 2015;12 (7):387-397. doi:10.1038/nrcardio.2015.39

7. Deshmukh A, Kumar G, Pant S, Rihal C, Murugiah K, Mehta JL. Prevalence of Takotsubo cardiomyopathy in the United States. $\mathrm{Am}$ Heart J. 2012;164(1):66-71 e1. doi:10.1016/j.ahj.2012.03.020

8. Schneider B, Athanasiadis A, Stollberger C, et al. Gender differences in the manifestation of tako-tsubo cardiomyopathy. Int $J$ Cardiol. 2013;166(3):584-588. doi:10.1016/j.ijcard.2011.11.027

9. Topal Y, Topal H, Dogan C, Tiryaki SB, Biteker M. Takotsubo (stress) cardiomyopathy in childhood. Eur J Pediatr. 2020;179 (4):619-625. doi:10.1007/s00431-019-03536-z

10. Sharkey SW, Windenburg DC, Lesser JR, et al. Natural history and expansive clinical profile of stress (tako-tsubo) cardiomyopathy. J Am Coll Cardiol. 2010;55(4):333-341. doi:10.1016/j.jacc.2009.08.057 
11. Urbinati A, Pellicori P, Guerra F, Capucci A, Clark AL. Takotsubo syndrome in the paediatric population: a case report and a systematic review. J Cardiovasc Med. 2017;18(4):262-267. doi: $10.2459 / \mathrm{JCM} .0000000000000446$

12. Singh K, Carson K, Usmani Z, Sawhney G, Shah R, Horowitz J. Systematic review and meta-analysis of incidence and correlates of recurrence of takotsubo cardiomyopathy. Int $J$ Cardiol. 2014;174(3):696-701. doi:10.1016/j.ijcard.2014.04.221

13. El-Battrawy I, Santoro F, Stiermaier T, et al. Incidence and clinical impact of recurrent Takotsubo syndrome: results from the GEIST registry. J Am Heart Assoc. 2019;8(9):e010753. doi:10.1161/JAHA.118.010753

14. Kato K, Di Vece D, Cammann VL, et al. Takotsubo Recurrence: morphological Types and Triggers and Identification of Risk Factors. J Am Coll Cardiol. 2019;73(8):982-984. doi:10.1016/j. jacc.2018.12.033

15. Arcari L, Cacciotti L, Limite LR, et al. Clinical characteristics of patients with takotsubo syndrome recurrence: an observational study with long-term follow-up. Int J Cardiol. 2021;329:23-27. doi:10.1016/j.ijcard.2020.12.047

16. Ghadri J-R, Wittstein IS, Prasad A, et al. International expert consensus document on Takotsubo syndrome (part I): clinical characteristics, diagnostic criteria, and pathophysiology. Eur Heart J. 2018;39(22):2032-2046. doi:10.1093/eurheartj/ehy076

17. Giavarini A, Chedid A, Bobrie G, Plouin PF, Hagege A, Amar L. Acute catecholamine cardiomyopathy in patients with phaeochromocytoma or functional paraganglioma. Heart. 2013;99 (19):1438-1444. doi:10.1136/heartjnl-2013-304073

18. Finsterer J, Stöllberger C. Central nervous system (CNS) disease triggering Takotsubo syndrome. International Cardiovascular Forum Journal. 2016;5. doi:10.17987/icfj.v5i0.183

19. Suzuki H, Matsumoto Y, Kaneta T, et al. Evidence for brain activation in patients with takotsubo cardiomyopathy. Circ J. 2014;78(1):256-258. doi:10.1253/circj.cj-13-1276

20. Abraham J, Mudd JO, Kapur NK, Klein K, Champion HC, Wittstein IS. Stress cardiomyopathy after intravenous administration of catecholamines and beta-receptor agonists. $\mathrm{J} \mathrm{Am} \mathrm{Coll}$ Cardiol. 2009;53(15):1320-1325. doi:10.1016/j.jacc.2009.0 2.020

21. Wittstein IS, Thiemann DR, Lima JA, et al. Neurohumoral features of myocardial stunning due to sudden emotional stress. $N$ Engl J Med. 2005;352(6):539-548. doi:10.1056/NEJMoa043046

22. Kume T, Kawamoto T, Okura H, et al. Local release of catecholamines from the hearts of patients with tako-tsubo-like left ventricular dysfunction. Circ J. 2008;72(1):106-108. doi:10.1253/circj.72.106

23. Ortak J, Khattab K, Barantke M, et al. Evolution of cardiac autonomic nervous activity indices in patients presenting with transient left ventricular apical ballooning. Pacing Clin Electrophysiol. 2009;32(Suppl 1):S21-5. doi:10.1111/j.15408159.2008.02221.x

24. Burgdorf C, von Hof K, Schunkert H, Kurowski V. Regional alterations in myocardial sympathetic innervation in patients with transient left-ventricular apical ballooning (Tako-Tsubo cardiomyopathy). $J$ Nucl Cardiol. 2008;15(1):65-72. doi:10.1016/j.nuclcard.2007.08.005

25. Komesaroff PA, Esler MD, Sudhir K. Estrogen supplementation attenuates glucocorticoid and catecholamine responses to mental stress in perimenopausal women. J Clin Endocrinol Metab. 1999;84(2):606-610. doi:10.1210/jcem.84.2.5447

26. Sung BH, Ching M, Izzo JL, Dandona P, Wilson MF. Estrogen improves abnormal norepinephrine-induced vasoconstriction in postmenopausal women. $J$ Hypertens. 1999;17(4):523-528. doi:10.1097/00004872-199917040-00010
27. Ghadri JR, Wittstein IS, Prasad A, et al. International Expert Consensus Document on Takotsubo Syndrome (Part I): clinical Characteristics, Diagnostic Criteria, and Pathophysiology. Eur Heart J. 2018;39(22):2032-2046. doi:10.1093/eurheartj/ ehy076

28. Kurisu S, Inoue I, Kawagoe T, et al. Time course of electrocardiographic changes in patients with tako-tsubo syndrome: comparison with acute myocardial infarction with minimal enzymatic release. Circ J. 2004;68(1):77-81. doi:10.1253/circj.68.77

29. Akashi YJ, Musha H, Nakazawa K, Miyake F. Plasma brain natriuretic peptide in takotsubo cardiomyopathy. QJM. 2004;97 (9):599-607. doi:10.1093/qjmed/hch094

30. Nef HM, Mollmann H, Hilpert P, et al. Sympathoadrenergic overstimulation in Tako-Tsubo cardiomyopathy triggered by physical and emotional stress. Int $J$ Cardiol. 2008;130(2):266-268. doi:10.1016/j.ijcard.2007.05.119

31. Pilgrim TM, Wyss TR. Takotsubo cardiomyopathy or transient left ventricular apical ballooning syndrome: a systematic review. Int $J \quad$ Cardiol. $\quad 2008 ; 124(3): 283-292 . \quad$ doi:10.1016/j. ijcard.2007.07.002

32. Pirlet C, Pierard L, Legrand V, Gach O. Ratio of high-sensitivity troponin to creatine kinase-MB in takotsubo syndrome. Int J Cardiol. 2017;243:300-305. doi:10.1016/j.jicard.2017.05.107

33. Dagrenat C, Von Hunolstein JJ, Matsushita K, et al. Value of Cardiac Biomarkers in the Early Diagnosis of Takotsubo Syndrome. J Clin Med. 2020;9(9):2985. doi:10.3390/ jcm9092985

34. Fröhlich GM, Schoch B, Schmid F, et al. Takotsubo cardiomyopathy has a unique cardiac biomarker profile: NT-proBNP/myoglobin and NT-proBNP/troponin $\mathrm{T}$ ratios for the differential diagnosis of acute coronary syndromes and stress induced cardiomyopathy. Int $J$ Cardiol. 2012;154(3):328-332. doi:10.1016/j.ijcard.2011.09.077

35. Pelliccia F, Parodi G, Greco C, et al. Comorbidities frequency in Takotsubo syndrome: an international collaborative systematic review including 1109 patients. Am J Med. 2015;128 (6):654e11-9. doi:10.1016/j.amjmed.2015.01.016

36. Gopalakrishnan P, Zaidi R, Sardar MR. Takotsubo cardiomyopathy: pathophysiology and role of cardiac biomarkers in differential diagnosis. World J Cardiol. 2017;9(9):723-730. doi:10.4330/wjc.v9. i 9.723

37. Citro R, Okura H, Ghadri JR, et al. Multimodality imaging in takotsubo syndrome: a joint consensus document of the European Association of Cardiovascular Imaging (EACVI) and the Japanese Society of Echocardiography (JSE). Eur Heart $J$ Cardiovascular Imaging. 2020;21(11):1184-1207. doi:10.1093/ehjci/jeaa149

38. Elesber AA, Prasad A, Bybee KA, et al. Transient cardiac apical ballooning syndrome: prevalence and clinical implications of right ventricular involvement. $J$ Am Coll Cardiol. 2006;47 (5):1082-1083. doi:10.1016/j.jacc.2005.12.004

39. Tamborini G, Piazzese C, Lang RM, et al. Feasibility and Accuracy of Automated Software for Transthoracic Three-Dimensional Left Ventricular Volume and Function Analysis: comparisons with Two-Dimensional Echocardiography, Three-Dimensional Transthoracic Manual Method, and Cardiac Magnetic Resonance Imaging. $\quad J \quad A m \quad$ Soc Echocardiogr. 2017;30(11):1049-1058. doi:10.1016/j.echo.2017.06.026

40. Nagata Y, Wu VC, Kado Y, et al. Prognostic Value of Right Ventricular Ejection Fraction Assessed by Transthoracic 3D Echocardiography. Circ Cardiovasc Imaging. 2017;10(2): e005384. doi:10.1161/CIRCIMAGING.116.005384

41. Hwang HJ, Sohn IS. A case of biventricular involvement of Takotsubo cardiomyopathy: 3D echocardiographic imaging. $J$ Echocardiogr. 2014;12(1):48-49. doi:10.1007/s12574-013-0200-2 
42. Prasad A, Lerman A, Rihal CS. Apical ballooning syndrome (Tako-Tsubo or stress cardiomyopathy): a mimic of acute myocardial infarction. Am Heart J. 2008;155(3):408-417. doi:10.1016/j.ahj.2007.11.008

43. Napp LC, Cammann VL, Jaguszewski M, et al. Coexistence and outcome of coronary artery disease in Takotsubo syndrome. Eur Heart J. 2020;41(34):3255-3268. doi:10.1093/eurheartj/ ehaa210

44. Kato K, Kitahara H, Fujimoto Y, et al. Prevalence and Clinical Features of Focal Takotsubo Cardiomyopathy. Circ J. 2016;80 (8):1824-1829. doi:10.1253/circj.CJ-16-0360

45. Eitel I, von Knobelsdorff-brenkenhoff F, Bernhardt P, et al. Clinical characteristics and cardiovascular magnetic resonance findings in stress (takotsubo) cardiomyopathy. JAMA. 2011;306 (3):277-286. doi:10.1001/jama.2011.992

46. Lyon AR, Bossone E, Schneider B, et al. Current state of knowledge on Takotsubo syndrome: a Position Statement from the Taskforce on Takotsubo Syndrome of the Heart Failure Association of the European Society of Cardiology. Eur J Heart Fail. 2016;18(1):8-27. doi:10.1002/ejhf.424

47. Scantlebury DC, Prasad A. Diagnosis of takotsubo cardiomyopathy-Mayo Clinic criteria. Circ J. 2014;1:CJ-14-0859.

48. Ghadri JR, Cammann VL, Jurisic S, et al. A novel clinical score (InterTAK Diagnostic Score) to differentiate takotsubo syndrome from acute coronary syndrome: results from the International Takotsubo Registry. Eur J Heart Fail. 2017;19(8):1036-1042. doi:10.1002/ejhf.683

49. Kato K, Sakai Y, Ishibashi I, Himi T, Fujimoto Y, Kobayashi Y. Predictors of in-hospital cardiac complications in patients with Takotsubo syndrome. Heart Vessels. 2018;33(10):1214-1219. doi:10.1007/s00380-018-1172-y

50. Kato K, Lyon AR, Ghadri JR, Templin C. Takotsubo syndrome: aetiology, presentation and treatment. Heart. 2017;103 (18):1461-1469. doi:10.1136/heartjnl-2016-309783

51. Madias C, Fitzgibbons TP, Alsheikh-Ali AA, et al. Acquired long QT syndrome from stress cardiomyopathy is associated with ventricular arrhythmias and torsades de pointes. Heart Rhythm. 2011;8(4):555-561. doi:10.1016/j.hrthm.2010.12.012

52. Santoro F, Ieva R, Ferraretti A, et al. Hemodynamic Effects, Safety, and Feasibility of Intravenous Esmolol Infusion During Takotsubo Cardiomyopathy With Left Ventricular Outflow Tract Obstruction: results From A Multicenter Registry. Cardiovasc Ther. 2016;34(3):161-166. doi:10.1111/1755-5922.12182

53. Paur H, Wright PT, Sikkel MB, et al. High levels of circulating epinephrine trigger apical cardiodepression in a $\beta 2$-adrenergic receptor/Gi-dependent manner: a new model of Takotsubo cardiomyopathy. Circulation. 2012;126(6):697-706. doi:10.1161/ CIRCULATIONAHA.112.111591

54. De Santis V, Vitale D, Tritapepe L, Greco C, Pietropaoli P. Use of levosimendan for cardiogenic shock in a patient with the apical ballooning syndrome. Ann Intern Med. 2008;149(5):365-367. doi:10.7326/0003-4819-149-5-200809020-00028

55. Isogai T, Matsui H, Tanaka H, Fushimi K, Yasunaga H. Early $\beta$ blocker use and in-hospital mortality in patients with Takotsubo cardiomyopathy. Heart. 2016;102(13):1029-1035. doi:10.1136/ heartjnl-2015-308712

56. Kurisu S, Inoue I, Kawagoe T, et al. Incidence and treatment of left ventricular apical thrombosis in Tako-tsubo cardiomyopathy. Int $J$ Cardiol. 2011;146(3):e58-60. doi:10.1016/j.ijcard.20 08.12.208

57. Bietry R, Reyentovich A, Katz SD. Clinical management of takotsubo cardiomyopathy. Heart Fail Clin. 2013;9(2):177-186. doi:10.1016/j.hfc.2012.12.003

58. Kurisu S, Kihara Y. Clinical management of takotsubo cardiomyopathy. Circ J. 2014;78(7):1559-1566. doi:10.1253/ circj.CJ-14-0382
59. Ding KJ, Cammann VL, Szawan KA, et al. Intraventricular Thrombus Formation and Embolism in Takotsubo Syndrome: insights From the International Takotsubo Registry. Arterioscler Thromb Vasc Biol. 2020;40(1):279-287. doi:10.1161/ ATVBAHA.119.313491

60. Kurisu S, Inoue I, Kawagoe T, et al. Torsade de pointes associated with bradycardia and takotsubo cardiomyopathy. Can J Cardiol. 2008;24(8):640-642. doi:10.1016/s0828-282x(08)70653-6

61. Purvis JA, Cunningham EL, McGlinchey PG, Barr SH. Drugs, electrolytes and tako-tsubo cardiomyopathy: triple aetiology of acquired long QT syndrome and torsades de pointes. Ulster Med J. 2009;78(3):188-189.

62. Santoro F, Ieva R, Musaico F, et al. Lack of efficacy of drug therapy in preventing Takotsubo cardiomyopathy recurrence: a meta-analysis. Clin Cardiol. 2014;37(7):434 439. doi:10.1002/clc.22280

63. Brunetti ND, Santoro F, De Gennaro L, et al. Combined therapy with beta-blockers and ACE-inhibitors/angiotensin receptor blockers and recurrence of Takotsubo (stress) cardiomyopathy: a meta-regression study. Int $J$ Cardiol. 2017;230:281-283. doi:10.1016/j.ijcard.2016.12.124

64. Gelow J, Kruer M, Yadav V, Kaul S. Apical ballooning resulting from limbic encephalitis. Am J Med. 2009;122(6):583-586. doi:10.1016/j.amjmed.2008.12.016

65. Kakinuma Y, Kimura T, Sakae Y, Kubota S, Ono K, Kinno R. Takotsubo syndrome associated with autoimmune limbic encephalitis: a case report. BMC Cardiovasc Disord. 2021;21(1):86. doi:10.1186/s12872-020-01789-3

66. Samuels MA. The brain-heart connection. Circulation. 2007;116 (1):77-84. doi:10.1161/CIRCULATIONAHA.106.678995

67. Hiestand T, Hanggi J, Klein C, et al. Takotsubo Syndrome Associated With Structural Brain Alterations of the Limbic System. J Am Coll Cardiol. 2018;71(7):809-811. doi:10.1016/j.jacc.2017.12.022

68. Templin C, Hanggi J, Klein C, et al. Altered limbic and autonomic processing supports brain-heart axis in Takotsubo syndrome. Eur Heart J. 2019;40(15):1183-1187. doi:10.1093/eurheartj/ehz068

69. de Chazal HM, Del Buono MG, Keyser-Marcus L, et al. Stress cardiomyopathy diagnosis and treatment: JACC state-of-the-art review. J Am Coll Cardiol. 2018;72(16):1955-1971. doi:10.1016/ j.jacc.2018.07.072

70. Ghadri JR, Kato K, Cammann VL, et al. Long-Term Prognosis of Patients With Takotsubo Syndrome. J Am Coll Cardiol. 2018;72 (8):874-882. doi:10.1016/j.jacc.2018.06.016

71. Montone RA, Niccoli G, Del Buono MG, Galiuto L, Crea F. Emotional versus physical Takotsubo syndrome: two faces of the same medal or two different syndromes? Prog Cardiovasc Dis. 2020;S0033-0620(20):30142. doi:10.1016/j.pcad.2020.07.005

72. Ghadri JR, Sarcon A, Diekmann J, et al. Happy heart syndrome: role of positive emotional stress in takotsubo syndrome. Eur Heart J. 2016;37(37):2823-2829. doi:10.1093/eurheartj/ ehv757

73. Nayeri A, Rafla-Yuan E, Krishnan S, et al. Psychiatric Illness in Takotsubo (Stress) Cardiomyopathy: a Review. Psychosomatics. 2018;59(3):220-226. doi:10.1016/j.psym.2018.01.011

74. Summers MR, Lennon RJ, Prasad A. Pre-morbid psychiatric and cardiovascular diseases in apical ballooning syndrome (tako-tsubo/stress-induced cardiomyopathy): potential pre-disposing factors? J Am Coll Cardiol. 2010;55(7):700-701. doi:10.1016/j.jacc.2009.10.031

75. Corrigan III FE, Kimmel MC, Jayaram G. Four cases of takotsubo cardiomyopathy linked with exacerbations of psychiatric illness. Innov Clin Neurosci. 2011;8(7):50.

76. Yaqub Y, Jenkins LA, Nugent KM, Chokesuwattanaskul W. Postpartum depression and apical ballooning syndrome (takotsubo syndrome). J Obstet Gynaecol Can. 2009;31(8):736-739. doi:10.1016/S1701-2163(16)34279-7 
77. Maldonado JR, Pajouhi P, Witteles R. Case reports broken heart syndrome (takotsubo cardiomyopathy) triggered by acute mania: a review and case report. Psychosomatics. 2013;54(1):74-79. doi:10.1016/j.psym.2012.03.009

78. Christoph M, Ebner B, Stolte D, et al. Broken heart syndrome: tako Tsubo cardiomyopathy associated with an overdose of the serotonin-norepinephrine reuptake inhibitor Venlafaxine. Eur Neuropsychopharmacol. 2010;20(8):594-597. doi:10.1016/j. euroneuro.2010.03.009

79. Selke KJ, Dhar G, Cohn JM. Takotsubo cardiomyopathy associated with titration of duloxetine. Tex Heart Inst J. 2011;38 (5):573-576.

80. Naguy A, Al-Mutairi H, Al-Tajali A. Atomoxetine-related Takotsubo Cardiomyopathy. J Psychiatr Pract. 2016;22 (3):232-233. doi:10.1097/PRA.0000000000000152

81. Kitami M, Oizumi H, Kish SJ, Furukawa Y. Takotsubo cardiomyopathy associated with lithium intoxication in bipolar disorder: a case report. J Clin Psychopharmacol. 2014;34(3):410-411. doi:10.1097/JCP.0b013e3182a95a27

82. Narayanan A, Russell MD, Sundararaman S, Shankar KK, Artman B. Takotsubo cardiomyopathy following electroconvulsive therapy: an increasingly recognised phenomenon. BMJ Case Rep. 2014;2014:bcr2014206816. doi:10.1136/bcr-2014-206816

83. Nayeri A, Rafla-Yuan E, Farber-Eger E, et al. Pre-existing psychiatric illness is associated with increased risk of recurrent Takotsubo cardiomyopathy. Psychosomatics. 2017;58 (5):527-532. doi:10.1016/j.psym.2017.04.008

84. Burgdorf C, Kurowski V, Bonnemeier H, Schunkert H, Radke PW. Long-term prognosis of the transient left ventricular dysfunction syndrome (Tako-Tsubo cardiomyopathy): focus on malignancies. Eur J Heart Fail. 2008;10(10):1015-1019. doi:10.1016/j.ejheart.2008.07.008

85. Desai R, Abbas SA, Goyal H, et al. Frequency of Takotsubo Cardiomyopathy in Adult Patients Receiving Chemotherapy (from a 5-Year Nationwide Inpatient Study). Am J Cardiol. 2019;123(4):667-673. doi:10.1016/j.amjcard.2018.11.006

86. Desai A, Noor A, Joshi S, Kim AS. Takotsubo cardiomyopathy in cancer patients. Cardio-Oncology. 2019;5(1):1-16. doi:10.1186/ s40959-019-0042-9

87. Madias JE. What is/are the trigger (s) of takotsubo syndrome in cancer patients receiving chemotherapy? Int $J$ Cardiol. 2016;222:253. doi:10.1016/j.ijcard.2016.07.246

88. Kinno R, Kii Y, Uchiyama M, Owan Y, Yamazaki T, Fukui T. 5-fluorouracil-induced leukoencephalopathy with acute stroke-like presentation fulfilling criteria for recombinant tissue plasminogen activator therapy. J Stroke Cerebrovasc Dis. 2014;23 (2):387-389. doi:10.1016/j.jstrokecerebrovasdis.2013.01.014

89. van Kuilenburg AB. Dihydropyrimidine dehydrogenase and the efficacy and toxicity of 5-fluorouracil. Eur J Cancer. 2004;40 (7):939-950. doi:10.1016/j.ejca.2003.12.004

90. Knott K, Starling N, Rasheed S, et al. A case of Takotsubo syndrome following 5-fluorouracil chemotherapy. Int J Cardiol. 2014;177(2):e65-7. doi:10.1016/j.ijcard.2014.09.154
91. Stewart T, Pavlakis N, Ward M. Cardiotoxicity with 5-fluorouracil and capecitabine: more than just vasospastic angina. Intern Med J. 2010;40(4):303-307. doi:10.1111/j.1445-5994.2009.02144.x

92. Franco TH, Khan A, Joshi V, Thomas B. Takotsubo cardiomyopathy in two men receiving bevacizumab for metastatic cancer. Ther Clin Risk Manag. 2008;4(6):1367-1370. doi:10.2147/tcrm. s3960

93. Bhakta S, Flick SM, Cooney MM, et al. Myocardial Stunning Following Combined Modality Combretastatin-Based Chemotherapy: two Case Reports and Review of the Literature. Clin Cardiol. 2009;32(12):E80-E84. doi:10.1002/clc.20685

94. Ng KH, Dearden C, Gruber P. Rituximab-induced Takotsubo syndrome: more cardiotoxic than it appears? Case Reports. 2015;2015:bcr2014208203.

95. Numico G, Sicuro M, Silvestris N, et al. Takotsubo syndrome in a patient treated with sunitinib for renal cancer. $J$ Clin Oncol. 2012;30(24):e218-20. doi:10.1200/JCO.2012.42.4911

96. Geisler BP, Raad RA, Esaian D, Sharon E, Schwartz DR. Apical ballooning and cardiomyopathy in a melanoma patient treated with ipilimumab: a case of takotsubo-like syndrome. J Immunother Cancer. 2015;3(1):4. doi:10.1186/s40425-015-0048-2

97. Kato K, Cammann VL, Napp LC, et al. Prognostic impact of acute pulmonary triggers in patients with takotsubo syndrome: new insights from the International Takotsubo Registry. ESC Heart Fail. 2021;8(3):1924-1932. doi:10.1002/ehf2.13165

98. Mathew B, Villarreal D. Two unusual cases of Takotsubo cardiomyopathy presenting with sudden cardiac death. Am J Med Sci. 2009;337(6):473-475. doi:10.1097/MAJ.0b013e3181956f49

99. Brezina P, Isler CM. Takotsubo cardiomyopathy in pregnancy. Obstet Gynecol. 2008;112(2 Pt 2):450-452. doi:10.1097/ AOG.0b013e3181662cfe

100. Hassan S. Takotsubo syndrome triggered by acute coronary syndrome in a cohort of 20 patients: an often missed diagnosis. Int J Cardiol Res. 2015;2(2):28-33.

101. Sandoval Y, Januzzi JL, Jaffe AS. Cardiac troponin for the diagnosis and risk-stratification of myocardial injury in COVID-19: JACC Review Topic of the Week. J Am Coll Cardiol. 2020;76 (10):1244-1258. doi:10.1016/j.jacc.2020.06.068

102. Giustino G, Pinney SP, Lala A, et al. Coronavirus and Cardiovascular Disease, Myocardial Injury, and Arrhythmia: JACC Focus Seminar. J Am Coll Cardiol. 2020;76 (17):2011-2023. doi:10.1016/j.jacc.2020.08.059

103. Madjid M, Safavi-Naeini P, Solomon SD, Vardeny O. Potential Effects of Coronaviruses on the Cardiovascular System: a Review. JAMA Cardiol. 2020;5(7):831-840. doi:10.1001/ jamacardio. 2020.1286

104. Giustino G, Croft LB, Oates CP, et al. Takotsubo Cardiomyopathy in COVID-19. J Am Coll Cardiol. 2020;76(5):628-629. doi:10.1016/j.jacc.2020.05.068

105. Moady G, Atar S. Takotsubo syndrome during the COVID-19 pandemic, state-of -the- art review. CJC Open. 2021. doi:10.1016/ j.cjco.2021.05.011

\section{Publish your work in this journal}

The Journal of Multidisciplinary Healthcare is an international, peerreviewed open-access journal that aims to represent and publish research in healthcare areas delivered by practitioners of different disciplines. This includes studies and reviews conducted by multidisciplinary teams as well as research which evaluates the results or conduct of such teams or healthcare processes in general. The journal

covers a very wide range of areas and welcomes submissions from practitioners at all levels, from all over the world. The manuscript management system is completely online and includes a very quick and fair peer-review system. Visit http://www.dovepress.com/testimonials. php to read real quotes from published authors. 